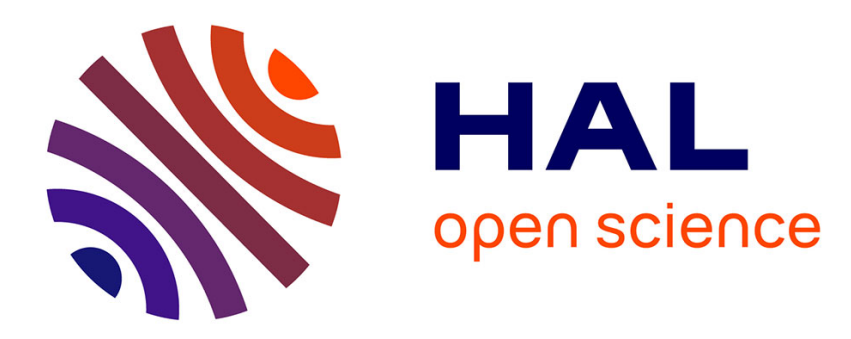

\title{
A short note on the complexity of computing strong pathbreadth
}

\author{
Guillaume Ducoffe
}

\section{To cite this version:}

Guillaume Ducoffe. A short note on the complexity of computing strong pathbreadth. Information Processing Letters, 2018, 133, pp.56-58. 10.1016/j.ipl.2018.01.005 . hal-01735826

\author{
HAL Id: hal-01735826 \\ https://hal.science/hal-01735826
}

Submitted on 16 Mar 2018

HAL is a multi-disciplinary open access archive for the deposit and dissemination of scientific research documents, whether they are published or not. The documents may come from teaching and research institutions in France or abroad, or from public or private research centers.
L'archive ouverte pluridisciplinaire HAL, est destinée au dépôt et à la diffusion de documents scientifiques de niveau recherche, publiés ou non, émanant des établissements d'enseignement et de recherche français ou étrangers, des laboratoires publics ou privés. 


\title{
A short note on the complexity of computing strong pathbreadth
}

\author{
Guillaume Ducoffe ${ }^{\mathrm{a}, \mathrm{b}}$ \\ ${ }^{a}$ ICI - National Institute for Research and Development in Informatics, Romania \\ ${ }^{b}$ The Research Institute of the University of Bucharest ICUB
}

\begin{abstract}
The strong pathbreadth of a given graph $G$ is the minimum $\rho$ such that $G$ admits a Robertson and Seymour's path decomposition where every bag is the complete $\rho$-neighbourhood of some vertex in $G$. We prove that deciding whether a given graph has strong pathbreadth at most one is NP-complete. The latter answers negatively to a conjecture of [Leitert and Dragan, COCOA'16].
\end{abstract}

Keywords: strong pathbreadth; path decomposition; acyclic clustering; graph theory; NP-complete.

\section{Introduction}

We refer to [4] for graph terminology. All graphs considered are finite and simple (hence, with neither loops nor multiple edges). In this paper, we solve for the first time the complexity of computing the strong pathbreadth of a given graph — that is a pathlike invariant first introduced in [13]. Roughly, a graph $G=(V, E)$ has strong pathbreadth at most $\rho$ if there exists a sequence $\mathcal{X}$ of balls of radius exactly $\rho$ in $G$ so that, for any vertex $v \in V$ (resp., for any edge $e=\{u, v\} \in E$ ), the balls in $\mathcal{X}$ that contain $v$ (resp. that both contain $u$ and $v$ ) induce a nonempty consecutive subsequence. This concept can be seen as a broad generalization of convex bipartite graphs: a wellknown class of graphs in algorithmic graph theory with strong pathbreadth equal to one [11].

Related work. Strong pathbreadth is part of the parameters expressible in the rich framework of acyclic clustering [9]. We first survey the known results about this framework. Formally, a Robertson and Seymour's tree decomposition $(T, \mathcal{X})$ of $G$ is a pair consisting of a tree $T$ and of a family $\mathcal{X}=\left(X_{t}\right)_{t \in V(T)}$ of subsets of $V$ indexed by the nodes of $T$ and satisfying: 
- $\bigcup_{t \in V(T)} X_{t}=V$

- for any edge $e=\{u, v\} \in E$, there exists a $t \in V(T)$ such that $u, v \in$ $X_{t}$

- for any $v \in V,\left\{t \in V(T) \mid v \in X_{t}\right\}$ induces a subtree, denoted by $T_{v}$, of $T$.

The sets $X_{t}$ are called the bags of the decomposition [16]. Furthermore, the length of $(T, \mathcal{X})$ is the minimum $D$ such that every bag $X_{t} \in \mathcal{X}$ has weak diameter $\max _{u, v \in X_{t}}$ dist $_{G}(u, v) \leq D$. The breadth of $(T, \mathcal{X})$ is the minimum $R$ such that every bag $X_{t} \in \mathcal{X}$ is included in the complete $R$-neighbourhood $N_{G}^{R}\left[v_{t}\right]=\left\{v \in V \mid \operatorname{dist}_{G}\left(v, v_{t}\right) \leq R\right\}$ of some vertex $v_{t} \in V$ ( $v_{t}$ may not be in the bag). An acyclic $(R, D)$-clustering of $G$ is a tree decomposition of $G$ with respective length and breadth no more than $D$ and $R$. Such decompositions can be used in order to obtain compact routing schemes and to solve approximately some distance related problems on graphs (e.g., see [1] and the papers cited therein). More recently, nontrivial relationships between the existence of acyclic $(R, D)$-clustering and the treewidth of a graph have been proved in [5]. The treewidth of a graph $G$ is the minimum $\omega$ such that $G$ admits a tree decomposition where all the bags have size at most $\omega+1$; many NP-hard problems on general graphs can be solved in quasi linear-time on bounded-treewidth graphs [2].

The complexity of computing acyclic $(R, D)$-clusterings has been studied recently. In particular, the treelength of a graph $G$ is the minimum $D$ such that it admits an acyclic $(D, D)$-clustering [6]. The treebreadth of $G$ is the minimum $R$ such that it admits an acyclic $(R, 2 R)$-clustering [7]. Similarly, the pathlength and the pathbreadth of $G$ are, respectively, the minimum length and breadth of a path decomposition of $G$ - with a path decomposition being any tree decomposition $(T, \mathcal{X})$ where $T$ is prescribed to be a path [8]. Computing any of these four above parameters is NP-hard [10, 15]. These negative results have motivated the study of a new optimization criterion, hopefully easier to compute. Namely, a tree decomposition $(T, \mathcal{X})$ of $G$ has strong breadth $\rho$ if every bag $X_{t} \in \mathcal{X}$ is the complete $\rho$-neighbourhood $X_{t}=N_{G}^{\rho}\left[v_{t}\right]=\left\{u \in V \mid \operatorname{dist}_{G}\left(u, v_{t}\right) \leq \rho\right\}$ for some vertex $v_{t} \in X_{t}$. The strong treebreadth of $G$ (resp., the strong pathbreadth of $G$ ) is the minimum $\rho$ such that $G$ admits a tree decomposition (resp., a path decomposition) of strong breadth $\rho$. Computing the strong treebreadth of a given graph $G$ is still NP-hard [13]. However, it was conjectured by the authors in [13] that 
the strong pathbreadth of a given graph can be computed in polynomial time. In this note, we disprove their conjecture.

More precisely, our main result is that deciding whether a graph has strong pathbreadth equal to one is NP-complete (Theorem 1). We do so by reducing from a relative of the CHORDAL SANDWICH problem [12]. Similar reductions have been used for proving the hardness of computing treelength, treebreadth and their path counterparts. However, we use different gadgets in our reduction than those presented in $[10,13,15]$. Furthermore, we use some properties that are specific to strong pathbreadth for proving the correctness of our reduction.

\section{The reduction}

Let $\operatorname{spb}(G)$ denote the strong pathbreadth of a given graph $G$. This whole section is devoted to prove the following result.

Theorem 1. Deciding whether $\operatorname{spb}(G)=1$ for a given graph $G$ is NPcomplete.

We need to introduce the following additional terminology and notations. First, we identify any path decomposition $(T, \mathcal{X})$ of $G$, where $T=$ $\left(t_{1}, t_{2}, \ldots, t_{k}\right)$ and $\mathcal{X}=\left(X_{i}\right)_{1 \leq i \leq k}$, with the sequence $\left(X_{1}, X_{2}, \ldots, X_{k}\right)$. A clique-path is a path decomposition where all the bags are cliques (inducing complete subgraphs), and a graph $G$ is an interval graph if and only if it has a clique-path [14]. In order to prove Theorem 1, we reduce from the InTERVALIZING COLOURED GRAPH problem (ICG), defined as follows:

Problem 1 (Intervalizing coloured Graph (ICG)).

Input: $A$ graph $G=(V, E)$; a colouring $c: V \rightarrow\{1, \ldots, k\}$.

Question: Is there an interval supergraph $H$ of $G$ which is properly coloured by $c$ ?

ICG is proved to be NP-complete already for $k=4$ (i.e., $c$ is 4-colouring) [3]. This constant upper-bound on the number of colours used will be a key element in our reduction.

Construction of the graph.. We now describe the reduction from ICG to the recognition of graphs with unit strong pathbreadth. Let $G=(V, E)$ be a graph and $c: V \rightarrow\{1, \ldots 4\}$ a proper 4-colouring of $G$. The graph $G^{\prime}=\left(V^{\prime}, E^{\prime}\right)$ is constructed from $G$ as follows. 
- Let $\mathcal{P}$ be the family of all vertex subsets $W \subseteq V$ such that, for every $u, v \in W$, we have $c(u) \neq c(v)$. Note that, since $c$ is a 4-colouring, any such subset $W$ has size $|W| \leq 4$. Hence, we have $|\mathcal{P}|=\mathcal{O}\left(n^{4}\right)$. Furthermore, let $K$ be a set containing a vertex $x_{W}$ for every subset $W \in \mathcal{P}$. We set $V^{\prime}=V \cup E \cup K$.

- We make of $K$ a clique of $G^{\prime}$. The other edges in $E^{\prime}$ are:

1. the edges $\{v, e\}$ for every $v \in V$ and for every $e \in E$ that is incident to $v$;

2. the edges $\left\{v, x_{W}\right\}$ for every $W \in \mathcal{P}$ and for every $v \in W$;

3. finally, the edges $\left\{e, x_{W}\right\}$ for every $W \in \mathcal{P}$ and for every $e=$ $\{u, v\} \in E$ such that $u, v \in W$.

The resulting graph $G^{\prime}$ can be constructed from $G$ in $\mathcal{O}\left(n^{4}\right)$-time.

Correctness.. The correctness of our reduction follows from the two lemmas that are presented next.

Lemma 2. For every $G=(V, E)$ and $c: V \rightarrow\{1, \ldots, 4\}$ being a yesinstance of ICG, the graph $G^{\prime}=\left(V^{\prime}, E^{\prime}\right)$ of the reduction satisfies $\operatorname{spb}\left(G^{\prime}\right)=$ 1.

Proof. Let $H$ be an interval supergraph of $G$ which is properly coloured by $c$ (such a supergraph exists by the hypothesis). Furthermore, let $\left(W_{1}, W_{2}, \ldots, W_{t}\right)$ be a clique-path of $H$. Observe that, since $H$ is properly coloured by $c$, we have that, for every $1 \leq i \leq t, W_{i} \in \mathcal{P}$. We claim that $\left(N_{G^{\prime}}\left[x_{W_{1}}\right], N_{G^{\prime}}\left[x_{W_{2}}\right], \ldots, N_{G^{\prime}}\left[x_{W_{t}}\right]\right)$ is a path decomposition of $G^{\prime}$. Since the vertices of $K$ are contained in all the neighbour sets $N_{G^{\prime}}\left[x_{W_{i}}\right], 1 \leq i \leq t$, we only need to verify that $\left(N_{G^{\prime}}\left[x_{W_{1}}\right] \backslash K, N_{G^{\prime}}\left[x_{W_{2}}\right] \backslash K, \ldots, N_{G^{\prime}}\left[x_{W_{t}}\right] \backslash K\right)$ is a path decomposition of $G^{\prime} \backslash K$. For that, let us first observe that, for every $1 \leq i \leq t, N_{G^{\prime}}\left[x_{W_{i}}\right] \cap V=$ $W_{i}$. Since by the hypothesis $\left(W_{1}, W_{2}, \ldots, W_{t}\right)$ is a path decomposition of $G$, every vertex of $V$ is contained in a nonempty consecutive subsequence of bags. Furthermore, for every $e=\{u, v\} \in E$, there is a nonempty consecutive subsequence of bags that both contain $u$ and $v$, which is by construction the set of bags $N_{G^{\prime}}\left[x_{W_{i}}\right]$ containing vertex $e$. Then, we have that $e$ and its two neighbours $u$ and $v$ are contained in a common bag $N_{G^{\prime}}\left[x_{W_{i}}\right]$, for some $1 \leq i \leq t$. There are no other vertices and edges in $G^{\prime} \backslash K$. So, the claim is proved. It directly implies $\operatorname{spb}\left(G^{\prime}\right)=1$.

Lemma 3. If, given $G=(V, E)$ and $c: V \rightarrow\{1, \ldots, 4\}$, the graph $G^{\prime}$ of the reduction satisfies $\operatorname{spb}\left(G^{\prime}\right)=1$, then $G$ and $c$ is a yes-instance of ICG. 
Proof. Let $\left(X_{1}, X_{2}, \ldots, X_{t}\right)$ be a path decomposition of $G^{\prime}$ such that: every bag $X_{i}, 1 \leq i \leq t$, is the closed neighbourhood of some vertex of $G^{\prime}$; and the number of pairs $u, v \in V$ such that $\{u, v\} \in E$ and $u$ and $v$ are contained in a common bag is maximized. We claim that $\left(X_{1} \cap V, X_{2} \cap V, \ldots, X_{t} \cap V\right)$ is a path decomposition of $G$. In order to prove it, it suffices to prove that, for every $e=\{u, v\} \in E$, there is bag that contains both $u$ and $v$. By contradiction, let $e=\{u, v\} \in E$ be such that $u$ and $v$ are not contained in a common bag. Since $\left(X_{1}, X_{2}, \ldots, X_{t}\right)$ is a path decomposition of $G^{\prime}$, vertex $e$ must be contained in a bag. Furthermore, we have $N_{G^{\prime}}[e]=\{u, v, e\} \cup\left\{x_{W} \in\right.$ $K \mid u, v \in W\}$. In particular, every vertex of $N_{G^{\prime}}[e] \backslash\{u, v\}$ is adjacent to $u$ and $v$. Hence, since every bag containing $e$ must be the closed neighbourhood of some vertex in $N_{G^{\prime}}[e]$, there must be two consecutive bags $X_{i}, X_{i+1}$ such that $\left\{X_{i}, X_{i+1}\right\}=\left\{N_{G^{\prime}}[u], N_{G^{\prime}}[v]\right\}$. However, in this situation $X_{i} \cap X_{i+1}=$ $\{e\} \cup\left\{x_{W} \in K \mid u, v \in W\right\}=N_{G^{\prime}}[e] \backslash\{u, v\}$. Thus, we could add a new bag $Y=N_{G^{\prime}}[e]$ between $X_{i}$ and $X_{i+1}$, that would increase the number of pairs $u^{\prime}, v^{\prime} \in V$ such that $\left\{u^{\prime}, v^{\prime}\right\} \in E$ and $u^{\prime}$ and $v^{\prime}$ are contained in a common bag. The latter contradicts the maximality of the path decomposition with respect to this property, so, $u$ and $v$ must be contained in a common bag. It proves, as claimed, that $\left(X_{1} \cap V, X_{2} \cap V, \ldots, X_{t} \cap V\right)$ is a path decomposition of $G$. Furthermore, by construction we have that for every two vertices $u, v \in V$ such that $c(u)=c(v)$, there can be no common neighbour of $u$ and $v$ in $G^{\prime}$. As a result, $X_{i} \cap V \in \mathcal{P}$ for every $1 \leq i \leq t$.

Finally, let $H=\left(V, \bigcup_{i=1}^{t}\left\{\{u, v\} \mid u, v \in X_{i}\right\}\right)$. It follows from the above that $H$ is an interval supergraph of $G$ and that $c$ is a proper colouring of $H$. Thus, $G$ and $c$ is a yes-instance of ICG.

For every graph $G=(V, E)$ and $c: V \rightarrow\{1, \ldots, 4\}$, let $G^{\prime}=\left(V^{\prime}, E^{\prime}\right)$ the graph obtained with our reduction. Combining Lemmas 2 and 3, we have $\operatorname{spb}\left(G^{\prime}\right)=1$ if and only if $G$ and $c$ is a yes-instance of ICG. Since ICG is NP-complete and that the graph $G^{\prime}$ of the reduction can be constructed in polynomial time, it proves Theorem 1.

\section{Acknowledgments}

We wish to thank the referees for their careful reading of the first version of this manuscript and their useful comments. 


\section{References}

[1] Abu-Ata, M., Dragan, F. F., 2016. Metric tree-like structures in realworld networks: an empirical study. Networks 67 (1), 49-68.

[2] Bodlaender, H., 2006. Treewidth: Characterizations, applications, and computations. In: WG 2006, Bergen, Norway. pp. 1-14.

URL http://dx.doi.org/10.1007/11917496_1

[3] Bodlaender, H. L., de Fluiter, B., 1996. On intervalizing k-colored graphs for dna physical mapping. Discrete Applied Mathematics 71 (1$3), 55-77$.

[4] Bondy, J. A., Murty, U. S. R., 2008. Graph theory. Grad. Texts in Math.

[5] Coudert, D., Ducoffe, G., Nisse, N., 2016. To approximate treewidth, use treelength! SIAM Journal of Discrete Mathematics 30 (3), 14241436 .

[6] Dourisboure, Y., Gavoille, C., 2007. Tree-decompositions with bags of small diameter. Discrete Mathematics 307 (16), 2008-2029.

[7] Dragan, F., Köhler, E., 2014. An approximation algorithm for the tree tspanner problem on unweighted graphs via generalized chordal graphs. Algorithmica 69 (4), 884-905.

[8] Dragan, F., Köhler, E., Leitert, A., 2014. Line-distortion, bandwidth and path-length of a graph. In: Algorithm Theory-SWAT 2014. Springer, pp. 158-169.

[9] Dragan, F. F., Lomonosov, I., 2007. On compact and efficient routing in certain graph classes. Discrete applied mathematics 155 (11), 14581470 .

[10] Ducoffe, G., Legay, N., Nisse, N., 2016. On the complexity of computing treebreadth. In: IWOCA 2016 - 27th International Workshop on Combinatorial Algorithms. pp. 3-15.

[11] Glover, F., 1967. Maximum matching in a convex bipartite graph. Naval Research Logistics (NRL) 14 (3), 313-316.

[12] Golumbic, M., Kaplan, H., Shamir, R., 1995. Graph sandwich problems. Journal of Algorithms 19 (3), 449-473. 
[13] Leitert, A., Dragan, F., 2016. On strong tree-breadth. In: International Conference on Combinatorial Optimization and Applications. Springer, pp. 62-76.

[14] Lekkeikerker, C., Boland, J., 1962. Representation of a finite graph by a set of intervals on the real line. Fundamenta Mathematicae 51 (1), $45-64$.

[15] Lokshtanov, D., 2010. On the complexity of computing treelength. Discrete Applied Mathematics 158 (7), 820-827.

[16] Robertson, N., Seymour, P. D., 1984. Graph minors. III. planar treewidth. Journal of Combinatorial Theory, Series B 36 (1), 49-64. 